\title{
Ein Wiener Feuilletonist geht auf Urlaub. Ludwig Hevesis Die Althofleute. Ein Sommerroman (1897)
}

\section{Endre Hárs}

\section{Neohelicon}

Acta comparationis litterarum universarum

ISSN 0324-4652

Neohelicon

DOI 10.1007/s11059-018-0430-4
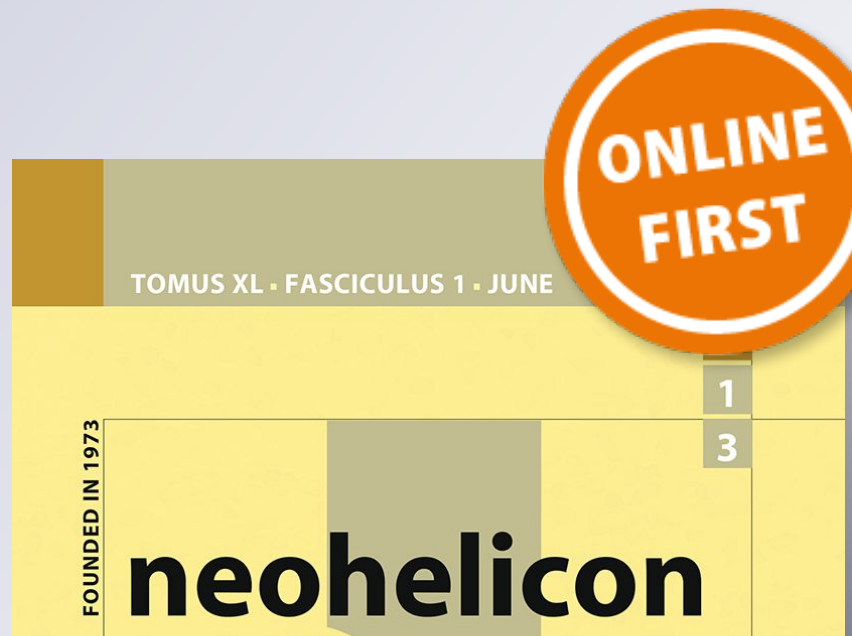

ACTA COMPARATIONIS

LITTERARUM UNIVERSARUM

TEXTUS ADULTER
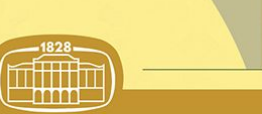

AKADÉMIAI KIADÓ

Springer 
Your article is protected by copyright and all rights are held exclusively by Akadémiai Kiadó, Budapest, Hungary. This e-offprint is for personal use only and shall not be selfarchived in electronic repositories. If you wish to self-archive your article, please use the accepted manuscript version for posting on your own website. You may further deposit the accepted manuscript version in any repository, provided it is only made publicly available 12 months after official publication or later and provided acknowledgement is given to the original source of publication and a link is inserted to the published article on Springer's website. The link must be accompanied by the following text: "The final publication is available at link.springer.com". 


\title{
Ein Wiener Feuilletonist geht auf Urlaub. Ludwig Hevesis Die Althofleute. Ein Sommerroman (1897)
}

\author{
Endre Hárs ${ }^{1}$
}

(C) Akadémiai Kiadó, Budapest, Hungary 2018

\begin{abstract}
In his almost single voluminous prose text, the Viennese columnist Ludwig Hevesi (1843-1910) makes experiments with the genre specifics of the "humorous novel", and fixes his subject in the vacationer milieu at the turn of the century. A 'Summer freshness' provides the material for a "summer novel"-also as a compromise of an author of 'short forms' with complex plots. The novel offers a portrait of the Habsburg monarchy's society: the relationship network of (Austrian) innkeepers, (European) holidaymakers and (Italian) guest workers can be socio-historically and imagologically evaluated and operates as a small portrait of the 'big world'.
\end{abstract}

Keywords Novel of the Fin de Siècle · Feuilleton · Culture of Tourism

Der Wiener Feuilletonist Ludwig Hevesi (1843-1910) - ein Autor, „in welchem der österreichisch-ungarische Dualismus seine sympathische Verkörperung findet“ (Teuber 1886) - hat in seinem gesamten Schaffen nur zwei literarische Werke von größerem Format veröffentlicht. Auf seinen recht erfolgreichen Jugendroman Jelky András bajai fiú rendkívüli kalandjai ötödfél világrészben (1872, dt. Des Schneidergesellen Andreas Jelky Abenteuer in vier Welttheilen, 1875) ließ er erst 1897 den zweiten und letzten Versuch, Die Althofleute. Ein Sommerroman folgen. Dabei erweist sich das so alleinstehende Werk gleich in seinem Untertitel als innovativ. Die Bezeichnung 'Sommerroman' ist in der zeitgenössischen Literatur über

Endre Hárs

hars@lit.u-szeged.hu

1 Institut für Germanistik, Universität Szeged, Szeged, Ungarn 
Sommerfrischen $^{1}$ alles andere als geläufig und begegnet erst ab Ende der 1920er Jahre öfter. ${ }^{2}$ Als Paratext ist er sogleich auf ein Spiel mit Bedeutungen angelegt: Die Spezifikation 'Sommer'kann sich zum einen auf den Rahmen der Handlung, den Chronotopos des Romans beziehen, zum anderen auf dessen Beschaffenheit als auf etwas für diese Jahreszeit Typisches hinweisen. Tatsächlich führt Die Althofleute zum einen ins Urlaubermilieu und beruht zum anderen auf einer Dreiecksgeschichte, einem 'Roman'der Leidenschaften—mit gutem Ausgang, wie es sich für eine Sommerlektüre geziemt. Für die folgende Analyse ist Hevesis Initiative in beiden Hinsichten von Relevanz. Der Roman erfasst ein zeittypisches Phänomen, die Institution der 'Sommerfrische', und wertet es literarisch aus. Mit dieser Option ist auch die Möglichkeit zu einem Porträt der Gesellschaft gegeben, das selbst dem scheinbar trivialen Plot Tiefe verleiht. Der Erweis dürfte für den Roman als solchen sprechen, dessen narrative Schichten die These von der Leichtigkeit als Sommerlektüre widerlegen.

\section{Sommer(frischen)roman}

Die Urlaubskultur der späten k. u. k. Monarchie ist Folge und zugleich Spiegel des sozialen Wandels des ausgehenden 19. Jahrhunderts. Sie zeigt, wie sich das aufkommende Bürgertum neue Lebens- und Repräsentationsräume verschafft. Im Rahmen dieser Entwicklung entsteht der Begriff der Freizeit, etabliert sich die Institution der Ferien (Lippmann 2016, 62), deren sich, je nach finanziellen Möglichkeiten, immer breitere bürgerliche Schichten bedienen. (Haas 1992, 367) Parallel zur Entstehung des modernen Tourismus findet eine Ausdifferenzierung der Reiseaktivitäten statt, mit Konsequenzen für die Infrastruktur der neuen Destinationen. In diesem Angebot von Möglichkeiten erscheint die 'Sommerfrische' als spezifischer Ort und Handlungsraum. Ihr Name ist mehrdeutig und bezeichnet sowohl den ,sommerliche[n] Aufenthalt[...] von Stadtbewohnern auf dem Land, im Gebirge oder am Meer" (Mai 2004, 8) als auch den geografischen Ort bzw. dessen Bauten, an dem bzw. in denen Urlaub gemacht wird. ${ }^{3}$ Das Spektrum der Sommerfrischenorte erstreckt sich von den größeren, vielbesuchten Kur- und Badeorten bis hin zu den kleineren, ländlichen Stätten und entfernt sich mit der Zeit mit Hilfe der neuen Eisenbahnverbindungen immer mehr vom großstädtischen Raum. (Haas 1992, 373) Die Bestimmung als Sommerfrische reicht von den Villen der Elite über das Hotelwesen bis hin zum privaten Angebot von Sommerwohnungen und ländlichen Funktionsgebäuden, wobei sich auch der soziale Status der Nutzer zwischen Villa und Provisorium entsprechend gliedert.

Der Boom von Sommerfrischen in der zweiten Hälfte des 19. Jahrhunderts steht im Einklang mit der großstädtischen Entwicklung. Die Sommerfrische verspricht

\footnotetext{
${ }^{1}$ Die Besprechung von Sommerfrischen ist üblich im zeitgenössischen Feuilleton; als literarischer und autobiografischer Schauplatz begegnet die Sommerfrische bei zahlreichen Zeitgenossen und Literaten. Vgl. Plath (Hg.) Plath 1999a, b; Tworek (Hg.) 2011.

2 Seit Otto Flakes Sommerroman (1927) bis in die Gegenwart begegnet die Titelgebung immer wieder.

3 Zur Etymologie des Begriffs Lippmann 2016, S. 166.
} 
Erholung vom Urbanen, und sei es auf klimatisch-therapeutische (Mai 2004, 10-12; Lippmann 2016, 187-204) oder auf kompensatorische Art. Insofern lassen sich der Kur- und der Ferienaufenthalt gar nicht strikt voneinander unterscheiden. (Mai 2004, 8) Die Sommerfrische ist zum einen imaginäre Konstruktion, zum anderen Umverwandlung eines konkreten Lebensraums in Wunschraum. Als Imagination „kommuniziert [sie] Entrücktheit, Selbstvergessenheit, Heimeligkeit und Geborgenheit. Er orientiert Handlungsprogramme wie den Nachmittagsspaziergang, das Frühstück auf der Veranda, das verträumte Liegen in der Hängematte oder das Blinzeln in die untergehende Sonne“ (Lippmann 2016, 169)_Aktivitäten, durch die sich die daran Beteiligten vom geschäftigen Alltag absetzen. Als konkretes Milieu entsteht die Sommerfrische „durch Bedeutungszuschreibungen an einen bestimmten Raum und durch bauliche Eingriffe in diesen Raum“ (Mai 2004, 17). Dessen wesentlichste Eigenart besteht darin, dass die umgebende Landschaft zum 'Mobiliar'des Großstädters wird. Man verschafft sich eine Umgebung urbaner Bedürfnisse und Komfortvorstellungen mit direktem Blick und Zugriff auf die 'Natur'. Auf diesem ,vorgeschobenen Stützpunkt der städtischen Zivilisation“ (Kos 1995, 9) agiert man, als wäre man deren Bindungen los. Dabei stehen die Urlaubsaktivitäten nach wie vor im Zeichen der sozialen Selbstverwirklichung und bleiben grundsätzlich auf die urbane Existenz bezogen. Der Aufenthalt in der Sommerfrische ist zum einen ein vom städtischen Milieu abgesetztes ,familiäres Vergnügen in einem durch Vertrauen charakterisierten Umfeld“ (Plath 1999a, b, 266), zum anderen Prestigehandlung: Man demonstriert dem in der Stadt zurückbleibenden 'Rest der Welt'bzw. der mitgereisten Öffentlichkeit von Urlaubern vor Ort, dass man sich den Ortswechsel für Tage bis Wochen leisten kann. Die Sommerfrischengesellschaft bewahrt ihren Sinn insofern als mise en abyme der städtischen.

Die Althofleute bedient sich insofern eines brandaktuellen Themas der Öffentlichkeit und der Medien. Der Schauplatz und die Figuren sind typisch für die Sommerfrischenkultur des mittleren Bürgertums (Haas 1992, 373-374) und werden in dieser Eigenschaft karikaturistisch überzeichnet. Hevesi präsentiert ein Kaleidoskop von Orts- und Figurenmerkmalen als wäre die Gesamtheit aller Orte und Urlauber an diesem einen Schauplatz versammelt. In der Lokalisierung des „Althofs“, einer im Waldbereich gelegenen bäuerlichen Wirtschaft, operiert er mit geografischen Namen, die teils real, teils fingiert sind und letztendlich den Eindruck vermitteln, dass der Althof überall liegen könnte. Immerhin stößt der homodiegetische Erzähler zum Auftakt auf eine typische und wie real klingende Zeitungsannonce:

„Ich las also das Wort »Sankt Ruprecht «. Der Verein für Hebung des Fremdenverkehrs in Sankt Ruprecht empfahl da sein Städtchen als empfehlenswerte Sommerfrische. 732 Meter über dem adriatischen Meer ozonreiche Luft - Bezirksgericht - Provinzial-Irrenanstalt - Alpenansicht Tannenwald - drei Ärzte - Museum für Stroh- und Korbflechterei - bisher 
absolut cholerafrei - Riesenburg - Galgenberg - der Althof (ältester Hof im ganzen Bannwald) ..."4

Die Form und die Angaben entsprechen den zeittypischen Werbetexten (Plath 1999a, b, 265), sodass man sich leicht zur Recherche veranlasst fühlt. Nun entspricht aber das am plausibelsten erscheinende Sankt Ruprecht an der Raab (Steiermark) nicht den Erwartungen, ${ }^{5}$ erst recht nicht, wenn man die im Roman hinzukommenden zusätzlichen Informationen sammelt. Denn über die aus dem Werbetext zitierten Informationen hinaus hat „Sankt Ruprecht" auch einen „Photograph[en]“ (174), einen „fürstlichen Park“mit „Warmhaus“ (66), eine „Folterkammer“ (238) und in nächster Umgebung eine „Luisenruhe“, benannt nach der „Prinzessin Luise, von Livonia, oder Franconia...“, und nach deren Verlobungsgeschichte mit dem „Prinzen Teodoro“ (60). Entsprechend hilft bei der Lokalisierung auch die weitere Umgebung nicht weiter. Weder der Kiefernwald noch der Granit, noch die „Bannwälder Trachtenbilder“ (176) ermöglichen die geografische Identifizierung. Sicher ist nur, dass man sich nicht im „Salzburgischen“ $(139 ; 179)$ befindet, wohin eine der Figuren gleichsam wie ins Ausland auszuwandern gedenkt.

Ist durch diese narrative Strategie eine cisleithanische Topografie von Sommerfrischen hergestellt, so verhält es sich mit dem Althof in der Nähe von „Sankt Ruprecht" selbst nicht anders. Der Roman verfolgt die Entstehung einer Gastwirtschaft ganz, wie sie zeitgenössisch festgehalten wird. So berichtet Arthur Michelis bereits 1869 mit leichten Untertönen über die Umstände einer neu gegründeten Sommerfrische:

„Die Entstehungsgeschichte einer solchen Sommerfrische ist etwa die folgende. Zuerst entdeckt ein Maler, welche Fülle von landschaftlichen Reizen ein entlegenes Thal birgt, hört in der Schenke des nächsten Dörfleins, daß daselbst vor ihm schon ein angelnder Engländer verweilt hat, und wird dessen Zimmernachfolger. Dieser erste Pionier der Cultur hat am Fensterkreuz einen Nagel für seinen Rasierspiegel eingeschlagen, sonst ist Alles noch auf der Stufe, die unmittelbar nach der Pfahlbautenperiode eingetreten sein mag [...]. Mittlerweile züngelt die Cultur weiter und weiter, binnen fünf Jahren sind zwei lebensgefährliche Treppenstufen ausgebessert, auch die Wirthsleute haben gelernt, lesen und schreiben zwar noch nicht, wohl aber rechnen. Jener Nagel des Engländers war der erste zum Sarge ihrer Herzenseinfalt. Schon kommt es vor, daß sie gleich zum ersten Male einen nicht in ihrer Mundart ausgedrückten Satz verstehen. [...]“ (Michelis 1869, 108-109)

Auch der Althof verdankt die Umverwandlung in eine Sommerfrische der Missernte und dem gleichzeitigen Eintreffen von Mr. Reginald Wanton Hicks und dessen Familie, die „die Pension Althof gewissermaßen entdeckt, ja miterfunden“

\footnotetext{
${ }^{4}$ Hevesi 1897, S. 2; im Weiteren mit Seitenzahlen im Haupttext zitiert.

5 Vgl. Illustrierter Wegweiser der österreichischen Kurorte, Sommerfrischen und Winterstationen 1909, S. 131 .
} 
(84) haben. ${ }^{6}$ Zur Zeit der Handlung des Romans beherbergt die namentlich aus einer großen Wohnstube, einer Wohnküche und einem „Liebdingstübl“ (66) bestehende „Panxion“ (73, auch 180) des Florian Anderer - andere Räumlichkeiten wie Schlafgemächer werden nicht genannt - bereits sechzehn Gäste „zu zwei Gulden der Kopf und Tag“ (ebd.). Abweichend von dem von Michelis beschriebenen Muster ist der Althofbauer nicht auf sich selbst im ländlichen 'Zivilisationsprozess'angewiesen: Als „Impresario des Alten“ (81) betätigt sich sein Neffe Theodor Bachmüller - beim Spitznamen auch „Herr Angel“ (89) genannt - und tut alles, um es mit der im Vorjahr gegründeten „Pension »zur Morgensonne« “ (73) aufzunehmen. In der Folge gestaltet sich der Roman als 'Geschichte einer Sommerfrische'. Diese beginnt mit anfänglichen Innovationen bzw. Schwierigkeiten und endet epilogisch mit dem definitiven Aufblühen des Unternehmens. $\mathrm{Zu}$ den ersten Maßnahmen des Herrn Angel gehören unter anderem ,die Einführung lithografierter Rechnungen mit der Ansicht des Althofs“ (89), die „Anschaffung von Postkarten mit der nämlichen Vignette“ (ebd.), Besorgung von Konsumgütern für die Herrschaften, etwa von „französische[m] Champagner“ (129) und einer ,automatische[n] Wage“ (156):

So ein Automat, erklärte er [Herr Angel, E. H.], wie jetzt in jeder anständigen Pension zu finden und verschönere das Leben der Gäste wesentlich. Für drei Kreuzer, die man in den elegant vergoldeten Löwenrachen werfe, könne man jeden Tag genau erfahren, um wie viel Kilo man seit gestern unter dem Einfluß der stählernen Luft und vorzüglichen Verpflegung dieses Sommerasyls zugenommen habe. [...] Der Automat war der Held des Abends und Florian Anderer ging auf des Herrn Angel Einflüsterungen hin mit dem festen Entschluß schlafen, noch heuer auch einen Cognac-Automaten zu bestellen.“ (157)

Zum unternehmerischen Beginn gehören auch die kleinen Missgriffe und Fauxpas. Das geeignete Medium für die gastwirtschaftlichen Fehler ist einer der Gäste, „Frau Angelique von Trübezal, aus Böhmen, Besitzerin einer vielbesuchten Ruine daselbst" (86). Sie ist aus der "Branche"und als Gast das touristische Gegenstück zum fleißigen Herrn Angel. Ihr passieren die Unfälle, und sie ist es auch, die die Reklamation und die kritische Beurteilung von Wirt und Gästen als ihre Vergnügung und 'Mission'betrachtet:

„Der Herr Angel saß mit gerunzelter Stirne da und kaute tief innen an etwas Schwerem. Das Bewußtsein einer Krise begann in ihm aufzudämmern, er sah den Althof, seine geniale Schöpfung, in Gefahr. Eine leere Schwemm'[die Küche als Essbereich, E.H.], daran muß ein Grand Hotel zu Grunde gehen.

\footnotetext{
${ }^{6}$ Dies geschah, indem Mr. Wicks den auf dem Felde mähenden Althofbauer, Florian Anderer, mit folgender Bitte angesprochen hat: „Herr Bauer, was bezahlen wir Ihnen, daß Sie lassen uns schneiden Ihren Korn?"Nachdem der Bauer für „fuchz'g Kreuzer ein jed's auf'n Tag“ (84) eingewilligt hat, nahmen Mr. Hicks und Familie „Unterstand im Althof, um jeden Tag Feldarbeit zu machen. Sie legten Bannwälder Tracht an, aßen am Familientisch und fanden das alles überaus gesund und billig. " (ebd.) Zu „freundschaftlichen Bande[n]“zwischen Vermietern und Gastfamilien vgl. Plath Plath 1999a, b, 266.
} 
Ein scharfes Gekicher störte ihn. Es kam von Frau von Trübezal. Sie studierte zum Zeitvertreib die Speisekarte, deren Orthographie sie stets höchlich belustigte. Jetzt schlug sie eine helle Lache auf und hielt sich die Seiten: » Das ist ausgezeichnet! Brillant! ... Was ist denn das für eine neue Speise, Herr Ang ... Pardon! Herr Bachmüller, wollt'ich sagen. « Sie schob ihm den Speisezettel hinüber, diesen Stolz des Herrn Angel, mit der schönen Ansicht des Althofs in Holzschnitt. In der That, da stand buchstäblich geschrieben: » Hamm und Ex.« “ (250)

In solchen Situationen wünscht sich Florian Anderer, er ,hätte doch lieber [...] als Bauer bei seinem Leisten bleiben [sollen]“ (167), und Herr Angel tut sein Bestes, um zu demonstrieren, ,wie man mit den Leuten reden muß[.] Wenig, aber g'scheit. Das imponiert ihnen und hebt das Geschäft.“ (222) Seine 'Politik'der Gastwirtschaft fordert jedenfalls vom Personal einiges, müssen sich doch die Einheimischen anstrengen, die Dienstleistungen des Hauses nach Herrn Angels Instruktionen und den Wünschen der Frau von Trübezal gemäß anzubieten (z. B. mit den städtischen Herrschaften hochdeutsch zu reden).

Der Althof wechselt im Späteren - eingebettet in die Geschichte und in Verbindung mit anderen Handlungssträngen - den Besitzer, erhält durch 'Einheiratung 'eine 'Finanzspritze'und blüht ganz und gar auf. Bei seinen wiederholten Besuchen trifft der Erzähler in der ,aufstrebende[n] Sommerfrische“ (376) eine deutliche Erhöhung des Komforts vor: „Bäder im Hause“, „Telegraph“, „Telephon“, „Wasserleitung mit Warm und Kalt“, „,die » gelesensten Zeitungen des Inund Auslandes«" (370) und Neubauten: ein „Lusthaus“ (359) bzw. eine „neuerbaute hochelegante Dépendance“ (356). Eine weitere Neuerung stellen „die echten Bauernspeisen“dar: ,[J]eden Tag eine, und die Städter fanden es ungeheuer ethnographisch, heute Grammelsterz und morgen Eierhaber zu naschen “ (370). Dass diese Entwicklung dennoch in kein Handbuch des Fremdenverkehrs gehört und mit durchgehender - wenngleich wohlwollender - Ironie in einem Roman angebracht ist, illustriert zum Schluss die Geschichte von dem „MesserAutomat[en]“ (390): Die Idee, durch Einwurf von drei Kreuzern aus einem zu diesem Zweck deponierten Gerät ein Messer zu erhalten, mit dem verliebte Herzen auf der Luisenhöhe ihre Namen in die Bäume schnitzen können, demonstriert die Innovationsfreude des jungen Unternehmerpaars. Das raffinierte Gerät des „Uhrmacher[s] von Sankt Ruprecht, ein[es] geriebene[n] Mechanikus“ (389) scheitert eben nur daran, dass „,halt alle Leut'ihre eigenen Messer bei sich haben!“ (391) und folglich niemand die neue Dienstleistung in Anspruch nimmt. Insofern bleibt der ironisch-humoristische Gegensatz dessen, was sich die ländlichen Anbieter und die städtischen Gäste jeweils unter 'Komfort'vorstellen, bis zum zukunftsträchtigen Schluss der Geschichte des Althofprojekts erhalten. Selbst in der 'zweiten Generation'bekommt man es mit der Herausforderung der Tourismusstrategie zu tun. $^{7}$

\footnotetext{
7 Eigentlich wurde der Althof gleich im ersten Jahr zwangsversteigert: So weit haben es der Althofbauer und Herr Angel mit ihren Investitionen und dem Tagestarif gebracht. Auch Holzer-Franz flieht übrigens als Fuhrmann vor dem Ruin in die Gastwirtschaft: vgl. seine Klage als über „die verfluxten Eisenbahnen“ (180).
} 
Mit der hier kurz rekapitulierten 'Geschichte einer Sommerfrische'sind die Deutungsmöglichkeiten des Romans freilich noch nicht erschöpft. Die Althofleute bietet auch tiefreichendere Dimensionen der wiedergegebenen historischen Situation. Als besonderer Schauplatz des späten 19. Jahrhunderts sorgt der Althof für einen Handlungsrahmen und eine Figurenwelt, in dem bzw. in der die sozialen Konsequenzen der neuen Urlauberkultur erprobt werden. Hevesis literarische Sommerfrische ist eine Heterotopie im Sinne Hans-Christian Lippmanns, der Foucaults Begriff auf diese Institution überträgt:

„Die Sommerfrische als Reise- und Erlebnisraum bietet dem Ich bedeutungsvolle Stimmungen, Erlebnisse, Zeitgefühle und Werte einer idealen bürgerlichen Ordnung. [...] Nur über kultivierte Vorstellungen, Erlebnisse und Praktiken, d. h. eine spezifische Sozialisation, kann das Ich sich der Sommerfrischengesellschaft zugehörig fühlen. “8

Das Konfliktpotential des Romans besteht im besonderen Interesse am Aushandeln von Gesellschaftsregeln, auf die hin die Figuren im Althof - sowohl die städtischen als auch die einheimischen - soziale Grenzen überschreiten. Die Darstellung dieses Sachverhaltes bietet mehr als der Untertitel „Sommerroman“, gar die Gattungszuschreibung „humoristischer Roman“ (Traeger 1897, 1) verspricht. An diesem tiefer reichenden Profil hat der Schauplatz bzw. die tourismushistorische (und -kritische) Perspektive wesentlichen Anteil. Freilich wird die Konstellation durch zusätzliche Momente 'verkompliziert', die ebenfalls mit der Geschichte der k. u. k. Monarchie zu tun haben.

\section{Städter, Provinzler und Gastarbeiter}

Eine späte Erzählerreflexion verweist rückwirkend recht deutlich auf die Figurenund Handlungskonstellation des Romans: Der nach dem Urlaub nach Wien abreisende - und erst in den beiden Epilogen wieder in den Althof zurückkehrende Erzähler meint ,die Handlung voll mit allen diesen Fäden, ... roten, weißen, grünen, aber ohne jede Ahnung, wie sie sich knüpfen und lösen würden“ (351) hinter sich zu lassen bzw. mitzunehmen, je nachdem, ob sich die Bemerkung auf die noch bevorstehenden Geschehnisse am Ort oder metafiktional auf die zu schreibende Geschichte bezieht. Tatsächlich hat man es bei den Figuren statt zwei mit drei 'Parteien“zu tun (welche auch immer mit ,,rot“, „,weiß“und „grün“gemeint sind): Neben den Urlaubern und den Einheimischen gibt es auch noch italienische Gastarbeiter am Ort, die die Konstellation grundlegend verändern.

In der Konzipierung der Gruppe der Sommerfrischler scheint dasselbe Prinzip vorzuherrschen, das auch die geografische Bestimmung des Ortes kennzeichnet. So wie dieser überall liegen könnte, sind auch die Gäste von überall her. Neben der Familie von Mr. Hicks und neben Frau von Trübezal gibt es einen Herrn Dirner, „brustschwacher Lehrer aus Marburg“ (85), „eine Frau Direktorswitwe Steiger, nach anderen Steiner“ (86), eine Frau von Bauer aus Wien - „für Florian Anderer

\footnotetext{
${ }^{8}$ Lippmann 2016, 34; vgl. Foucault 1992.
} 
besonders schätzbar, weil [...] eine Pension in Österreich um jeden Preis auch eine » Wienerin « müsse aufweisen können“ (88). Darüber hinaus gibt es ,eine Reihe von norddeutschen Damen“ (85), die immer zusammen auftreten, sodass sie auch öfter schlicht „die Fünf“ (106) genannt werden, und einen „Herr[n] ohne Koffer“, „weil er sein Gepäck in lauter kleinen Päckchen mitführte. Er hatte den praktischen Grundsatz, mit nichts von Hause abzureisen und unterwegs das jeweilen [sic!] Nötige zu kaufen.“ (86) Zu den Gästen zählt von seinem Status her auch der Erzähler selbst, „Dr. Franz Schneller, Sekretär aus Wien“ (20), der von seinen narrativen Rechten viel Gebrauch macht: Er wird nicht nur im „Liebdingstübl“ (26), im Wohngemach des jüngst verstorbenen alten Althofbauers untergebracht (damit zum symbolischen Familienmitglied und zur einzigen Figur, deren konkrete Unterbringung am Althof genannt wird). Er wird auch zur Vertrauens- und Mittelsperson aller 'Parteien', die - anders als Frau von Trübezal, „,die eigentliche Zunge des Althofs“ (87) - frei recherchieren kann ${ }^{9}$ und in dieser Eigenschaft auch akzeptiert und sogar zu Rate gezogen wird. Seine Rolle als homodiegetischer Erzähler wird dabei immer wieder heterodiegetisch erweitert: Er bekommt mehr mit als seine physische Präsenz an den Orten erlaubt, und er beglaubigt dies - gar erst, wenn gelegentlich auf die Figuren fokalisiert wird - nicht immer. ${ }^{10} \mathrm{Zu}$ seiner 'Person'leisten auch die von Wilhelm Schulz (1865-1952) angefertigten Illustrationen des Romans ihren Beitrag: Von einem Porträt kann man zwar nicht sprechen, dennoch erkennt man auf den Bildern immer wieder die Physiognomie Ludwig Hevesis. ${ }^{11}$ Diese Identifizierung ist nicht zwingend, dürfte jedenfalls das Publikum beeinflusst haben. Darüber hinaus kann sie als Bestandteil des Fiktionalisierungsspiels des Romans betrachtet werden: So wie „Sankt Ruprecht“"fast"der realweltliche Ort ist, ist „Dr. Franz Schneller, Sekretär aus Wien“beinahe ein Alter Ego Ludwig Hevesis.

Auf Seiten der Einheimischen spielen neben den bereits erwähnten Wirtspersonen Fräulein Luis und der „Holzer-Franz“eine wichtige Rolle. Luis ist „die Nichte, sozusagen Tochter des Althofbauern“ (26), dennoch nicht ganz einheimisch, da sie „in Linz aufgewachsen“ (20) ist und von daher die Ausbildung „,im Pensionat“ (370) und ihr Hochdeutsch mitbringt. „,[D]er lustige Holzer-Franz, die freie Zunge der Schwemm““ (95), ist Fuhrmann, Anwerber und späterer Ehemann von Luis. Von Berufs wegen 'welterfahrener'und charismatischer Mensch ist er in der Handlung mehrfach derjenige, der Anschluss an die Gäste findet. Über die beiden Figuren hinaus besitzt der Althof ein bescheidenes Personal und hat immer auch einheimische Gäste, vor allem Fuhrleute, die zwar namentlich erwähnt, in die Handlung jedoch nur episodisch mit einbezogen werden. Das Gegenüber der beiden 'Parteien'ist im Althof auch räumlich umgesetzt, indem den Herrschaften ,,[a]uf der Huiseite“ (81), in der ehemaligen großen Wohnstube, gedeckt wird, und die zum Trinken einkehrenden Einheimischen „,[a]uf der Pfuiseite“ (96), in der alten Küche,

\footnotetext{
9 Insofern ist Frau von Trübezal eine Konkurrenzfigur des Erzählers. Zu den Motivationen des Erzählers vgl. S. 222; 223; 228; 319.

${ }^{10}$ Vgl. z.m B.: „Von [...] ihrem Gespräch mit Tonio erwähnte sie nichts; ich erfuhr das erst später.“ (155); „Was in der Nacht geschehen, wußte ich noch nicht; ich sollte es zehn Tage später durch Marcello erfahren." (326).
}

11 Vgl. S. 1; 331; 335; 351; 369. 
bedient werden. Die beiden Räumlichkeiten bzw. Figurengruppen sind weitaus nicht so strikt voneinander getrennt, wie diese funktionale Symbolik vorzugeben scheint. Die Gäste begeben sich praktisch täglich in die „Schwemm“ “(81), auf die Pfuiseite, wo es „schöner“ (97) ist. Davon unabhängig besteht die Verbindung zwischen den beiden Gruppen bzw. Räumen auch auf eine andere Weise: „[E]in sogenannter Leuchtofen, [...] welche die Küche von der Wohnstube scheidet“ (68) und dessen „Schuber“auf der Wohnstubenseite geöffnet werden kann, sorgt dafür, dass man sich gegenseitig hört - nicht ohne Kommentare von drüben, die die Handlung begleiten.

Zusätzliche Handlung und romantisches Konfliktpotential verschafft über diese Konstellation hinaus die dritte 'Partei'in der Geschichte des Althofs. In der Umgebung beschäftigt der Unternehmer Signor Bergonza italienische Steinarbeiter, die Granit spalten und abends in der Schwemm 'einkehren. Tagsüber hört man aus der Ferne die Hammerschläge des schönen Tonio Verga und seines Freundes, Anselmo Bettini bzw. des hässlichen Marcello Ruffiani, eines Einzelgängers. Verga und Ruffiani sind Feinde, dessen Grund sich im Laufe des Romans nur schrittweise erhellt: Es handelt sich um eine tragische Liebesgeschichte und den Hass zweier Familien in Carrara. Der Aufenthalt des begabten jungen Bildhauers Tonio im Österreichischen ${ }^{12}$ verdankt sich der Beschuldigung am Mord an Ridolfo Ruffiani, Geliebter seiner Schwester, Estella Verga, und Bruder des Marcello Ruffiani. Die Tat hat in Wahrheit Vater Verga begangen, für den Tonio die Schuld auf sich nimmt. Tonios Gestalt fasziniert zum einen alle weiblichen Gäste des Althofs, zum anderen erweckt er Verdacht. Vor allem Frau von Trübezal ermittelt und macht immer wieder Anspielungen auf Räubergeschichten. ${ }^{13}$ So sind die italienischen Figuren Gegenstand kritischer Fremdwahrnehmung ${ }^{14}$ und begehrtes Anderes: Hauch des Südens mit exotischen und erotischen Komponenten.

Dabei ist die Räubergeschichte auch als intertextuelle Anspielung wirksam. Das, was Tonio und Marcello verbindet und zu Feinden macht, ist Ridolfo, der Bruder von Letzterem und ehemaliger Freund von Ersterem - ein ebenso schöner Jüngling wie Tonio, von dem sich Marcello als hässlicher 'Bruder'gleichermaßen fasziniert und abgestoßen fühlt. Die ungewollte brüderliche Zuneigung findet ihren nachträglichen Erweis, als Tonios Unschuld klar wird. Marcello erkennt in Tonio

\footnotetext{
${ }^{12}$ Grund sind diplomatische Spannungen zwischen Österreich-Ungarn und Italien und deren Folge, „die verhältnißmäßige Lauheit, mit der [...] die Auslieferung italienischer Verbrecher seitens ÖsterreichUngarns betrieben wurde" (202-203).

13 ,[U]nter diesen italienischen Steinmetzen da herum [möge] mancher schlimme Gesell vorkommen. Es werde ja auch gemunkelt, daß etwas mit dem Tonio los sei. [...] Augen habe der Mensch, schon wie der reine Teufel. Schwarzes Feuer! Sie [Frau von Trübezal, E. H.] habe einmal einen Roman gelesen: » Abällino der große Bandit «. Oder es falle ihr der » Rinaldo Rinaldini Schinderhanno Orlandini « ein. Aber wahr sei wahr, ein bildschöner Räuberhauptmann [...]" (90-91). 'Räubertum'begegnet übrigens auch als Selbstzuschreibung. Tonio berichtet über den „Glaubenssatz"seines Vaters als eines „Calabresen“wie folgt: „Jeder Mensch hat im Leben das Recht auf zwei Schüsse. [...] Diese zwei Schüsse kann er thun, wann und zu welchem Zweck er will: als Räuber oder zur Blutrache, zu politischem Mord oder zu Selbstmord.“ (283).

14 „Das Tischgespräch drehte sich diesmal hauptsächlich um Tonio [...]. Er habe immer so seine heimlichen Wege. Überhaupt sei etwas Unheimliches an dem ganzen Mann. Wenn sie [die Frau Direktorswitwe, E. H.] ihn ansehe, müsse sie immer denken, er ... Und auf keinen Fall möchte sie ihm im Walde allein begegnen." (90).
} 
den 'Räuber aus Not', bereut seine Rachepläne und stirbt - wenngleich nur durch einen Unfall, den seine Gefühle mitverursacht haben. Tonio wird gefasst, vor Gericht gestellt und durch Luis'Zeugenaussage freigesprochen. Das Drama des „hübschen Räuber[s]“ (122) endet in harmlosem Nachklang auf Schillers physiognomisch-moralisierendes Sturm-und-Drang-Stück.

Konkret wird die Beteiligung der Italiener an der Handlung durch die Dreiecksgeschichte zwischen Franz, Luis und Tonio. Sie wird erzählerisch in umgekehrter Handlungsfolge vermittelt: Das erste, was der Erzähler - unterwegs zum Althof - zu Gesicht bekommt ist das junge Paar, Luis und Tonio: eine Österreicherin, in verschämt verheimlichter Verbindung mit einem Italiener. Erst im späteren Verlauf wird klar, dass der Holzer-Franz der erste Verehrer war und Luis von Tonio nur abgeworben wurde. Der epilogische Schluss zeigt die Wiederherstellung der früheren Beziehung zu Franz - eine Restituierung der nationalen Ordnung 'mit Abstrichen", die nicht restlos glücklich macht. Luis und Franz übernehmen den Althof und führen jedenfalls die Sommerfrische zu neuer Blüte. Die Dreiecksgeschichte hat die diegetische Funktion, die drei Figurengruppen miteinander in Beziehung zu setzen: Das Schicksal der Italiener wird zur Sache der Einheimischen und zu einer Angelegenheit, die die Gäste beschäftigt, allen voran den Erzähler selbst als Mittelsmann.

\section{Verwicklungen}

Die beschriebene Handlungs- und Figurenkonstellation ermöglicht mit ihren drei 'Parteien'eine Gestaltung des Romans, die durch Verknüpfung der einzelnen Elemente Komplexität herstellt. Die Geschichte der Sommerfrische verbindet sich mit einer Dorfgeschichte, der wiederum eine 'romantische'Geschichte aus dem Süden implementiert ist, sodass das jeweils Typische immer durch Atypisches überschrieben wird.

Die „Huiseite“ (die Urlaubergesellschaft) und die „Pfuiseite“ (die Dorfgemeinschaft) sind nicht isoliert, der Leuchtofen ist gleichsam Symbol des stattfindenden Austausches. Weder hier noch dort ist man jedoch nur mit dem einen bzw. dem anderen Kollektiv beschäftigt. So wie in der umgebenden Landschaft das Geräusch der Steinmetze widerhallt, ist im Althof ständig auch die dritte 'Partei'(die Gruppe der Italiener) präsent. Die aus der Wohnstube in die Schwemm'herüberkommenden Gäste verteilen sich an Tischen der (einheimischen) Fuhrleute und der (fremden) Steinarbeiter. Die Situationen werden immer 'zu dritt'ausgehandelt, das Mischverhältnis trägt zur Beseitigung des jeweiligen Problems bzw. zum weiteren Handlungsverlauf das Seine bei. Alles richtet sich darauf, die Ausnahmesituation des aus den drei Extremen zusammengesetzten Sommerfrischenkollektivs in den Griff zu bekommen und das soziale Regelwerk der Heterotopie zu begründen.

Gruppiert um jeweils einen zentralen Konflikt lassen sich vier kapitelübergreifende Episoden des Romans voneinander unterscheiden. In allen vier Fällen lässt sich das beschriebene Mischverhältnis der verschiedenen Anliegen, das Aushandeln von Meinungen und Handlungsmotivationen rekapitulieren. Schauplatz der ersten Konfliktepisode ist die Schwemm', in der sich gleich im Anschluss an die 
erzählerische Vorstellung der Gäste und des Althofpersonals alle zusammenfinden. Man beobachtet sich gegenseitig, wobei sich die Aufmerksamkeit schnell polarisiert: Während „Tonio und die Luis vor den Leuten wenig Notiz von einander n[e]hmen“ (99), wenden sich die Anwesenden je nachdem Luis oder Tonio zu: „Alles, was Mannsbild hieß, drückte sich irgendwie und irgendwann an die Luis heran und sagte ihr irgendwas Schönes“ (100). Umgekehrt nähern sich alle sich eintreffenden weiblichen Gäste dem italienischen Tisch:

Dort saßen Tonio, Anselmo und Filippo bei ihrem » Fiff « (Pfiff) und sagten kein Wort. Tonio an der äußeren Ecke. Dicht neben ihm hatte Monikali sich auf einen Holzsessel seinen Weidling mit Erdäpfeln hingestellt, die sie schälte, wenn sie nicht bedienen mußte. [...]

Dann kamen die Damen Hicks herein; das zierliche Mädchen in Bannwäldertracht, eine Rose am Brustlatz, die so schlecht befestigt war, daß sie schon während des Plätzesuchens - das allgemeine Aufstehen und Rücken machte etwas Gedränge - auf Tonios Tisch niederfiel.“ (103)

Nur der Erzähler und der Holzer-Franz folgen nicht diesem 'instinktiven'Muster und behalten den Mechanismus selbst und dessen Motor, das scheinbar ahnungslos wirkende junge Paar, im Auge. Franz - ,ohne Widerrede der schönste Fuhrmann im Bannwald“" (107) - lässt seinen Gefühlen durch kritische Anmerkungen und Spottlieder auf die Gesellschaft freien Lauf. Die höchste Spannung wird während des anschließenden 'harmlosen 'Gesellschaftsspiels erreicht. Der kurz abwesende Franz findet die Gesellschaft damit beschäftigt wieder, Luis mit verbundenen Augen erraten zu lassen, „ob's ein » Bua « oder ein » Mensch « sei“, der ihr ,mit einem Virginier-Stroh am » G'nack « kitzle“ (119). Luis errät jedesmal, ob ein Mann oder eine Frau sie berührt, bis Tonio an der Reihe ist, dem aber Holzer-Franz den Halm aus der Hand reißt, um es selbst zu probieren. Das Ergebnis ist fatal, tippt doch Luis auf „Dame“ "und entschuldigt ihren Fehler mit den Worten: „Na ja, wann eins aber auch gar so zuckerbretzelzärtlich mit'm Stroh da umfladert..." (123). Ihre Erklärungen erweisen sich in dieser doppelt peinlichen Situation mehr als „Zwecklos“ (ebd.), sodass die beiden ebenso überraschten wie verletzten Männer nun gleich einander „Aug in Auge“ (124) zu stehen kommen und Mr. Hicks das Fazit zieht: „Well! Lassen Sie uns haben etwas boxing!“ (ebd.).

Statt einer Prügelei begnügen sich die beiden jungen Männer jedoch mit einer Kräftedemonstration: Tonio hebt ein volles Bierfass, Franz Frau von Trübezal samt „schwere[m] eichene[m] Bauernstuhl“ (126) in die Höhe, wobei deren „Luftfahrt"einen komischen Effekt erzeugt und die gegenseitige Vermeidung von Gewalt eine Versöhnung bewirken kann. Der durch die Dreiecksgeschichte generierte Konflikt zwischen den Einheimischen und den 'Gastarbeitern'wird damit - bei Mr. Hicks Kommentar und Frau von Trübezals 'Körpereinsatz'- 'urlaubermäßig'aufgelöst. In dieser Gesellschaft geziemt es sich nicht, zur üblichen männlich-ländlichen Problemlösung zu greifen. In der Folge wird - passend zu einer aufkommenden Sommerfrische und zur großen Freude Herrn Angels - mit „einige[n] Flaschen französische[m] Champagner“ (129) gefeiert. Dabei wird auch die sexuelle Polarisierung der Schwemm'wird entschärft. Der Umtrunk mündet in 
einen Ball, in dessen karnevalistischem Wirbel die Rollenverteilung umgekippt wird und jeder mit jedem über soziale Grenzen hinweg in Berührung kommt:

Alles tanzte durch- und miteinander, auch ein paar Holzknechte, die das Hallo angelockt hatte. Der Holzer-Franz [...] trat [...] an Mrs. Hicks heran, verneigte sich wie ein Wiener und sagte sehr hochdeutsch:

»Gnädiche Frau, dürfte ich um das Vegnüchen der Auszeichnung bitten?«

Mrs. Hicks wurde rot, obgleich dies bei ihrer blühenden Gesichtsfarbe nicht leicht war, und sagte:

$\gg$ Sehr gerne, Mister Holzer. « [...]

Eine Polka des Mr. Hicks mit Monikali, welche noch in einer Hand einen halbgeschälten » Erdäpfel« und in der andern das Messer hielt, wurde dieserhalb für die Umgebung lebensgefährlich. Die mutigsten Männer boten alle Gewandtheit auf, um ihr, während sie vorbeihopste, die Mordwaffe zu entwinden. [...]

Anselmo und Filippo hatten ausdrücklich die fünf Freundinnen des Fräuleins Hahn » im Block « auf sich genommen und bemühten sich, sie » im Akkord aufzuarbeiten «.“(131-132)

Nur der Herr Ministerialsekretär macht nicht mit und begnügt sich damit, aus auktorialer Perspektive detailliert aufzuzeichnen, wie sich die drei 'Parteien'vermischen und die sozialen Differenzen - Körper an Körper - aufgehoben werden. Etwas 'Neues'entsteht, wenngleich im Ausnahmezustand des Abends und der Heterotopie.

Die zweite Episode mit einem Konflikt, dessen Entstehung und Auflösung dem sozialen Mischverhältnis des Althofs geschuldet ist, geht weniger versöhnlich aus. Hauptfigur ist hier Frau von Trübezal, die gern im Mittelpunkt steht und ihre Mitmenschen manipuliert. In dieser Episode verliert sie im Austausch der drei 'Parteien'die Kontrolle und trägt ungewollt und eigentlich auch unverdient die Rechnung davon. Der Konflikt beginnt damit, dass Frau von Trübezal in ihrer fremdenfeindlichen Räubergeschichte fortfährt, indem sie die Gesellschaft vielsagend auf einen Zeitungsbericht bezüglich der Auslieferung von Verbrechern zwischen Italien und Österreich-Ungarn aufmerksam macht. ${ }^{15}$ Als im weiteren Verlauf Nachricht von einer Anzeige gegen Tonio und einer Durchsuchung seiner Hütte kommt, steht Frau von Trübezal als Beschuldigte und Sündenbock da. In ihrer Verdächtigung tun sich nun Einheimische und Sommerfrischler zusammen: HolzerFranz seinerseits erfindet eine ortspezifische Überlieferung, die „G'schicht 'vom Armesünderlupfen“ (241), derzufolge unbestraften Missetaten - diesmal der Verleumdung - durch das Volk Prozess gemacht wird. Tatsächlich wird die alte Richtstätte am Galgenberg von Tag zu Tag von unsichtbaren Händen neu aufgerichtet - ein Rätsel, dem Frau von Trübezal als geübte Detektivin dennoch schnell auf die Schliche kommt. Trotzdem hat ihre Beschuldigung auch in der Sommerfrischengesellschaft Konsequenzen. Die kleinen Sticheleien und Unannehmlichkeiten, mit denen sie sonst alle belästigt, führen diesmal zum allgemeinen Aufbegehren gegen sie. In der Folge verabschiedet sich eine Figur aus dem Althof

15 S. 202-203. 
und aus der Handlung, um erst wieder im ersten Epilog - nach einer Bußprozession aller, unter feierlichen Umständen - in den Althof wieder einzuziehen. Denn zum Schluss weiß man, dass die Anzeige gegen Tonio Marcellos Werk war und Frau von Trübezal keine Schuld außer ihrer Indiskretionen trägt, mit denen man zum Schluss gleichsam vertraut umgeht. Die Episode präsentiert zum einen eine handlungsbezogene Verwicklung von Motivationen - italienischer Rachezug, Frust der Sommerfrischler, Solidarität von Einheimischen -, zum anderen ein wiederholtes Zusammenwirken des Sommerfrischenkollektivs über soziale Grenzen hinweg. Nur beruht die heterotopische Meinungsbildung diesmal nicht auf dem Karneval, sondern auf dem Sündenbock und hinterlässt in dieser Eigenschaft nachteilige Spuren in der kleinen Gesellschaft. Das zeitweilige Verschwinden einer der bestimmenden Sommerfrischlerfiguren verblüfft als narrative Lösung und eröffnet für die nächste Episode, für die Eskalation der Dreiecksgeschichte Freiraum. Erst nach deren Lösung, in den Epilogen, kann die erzählerische Rückkehr zur Sommerfrischengesellschaft und eine Wiederherstellung von deren Ordnung erfolgen.

Die dritte konfliktreiche Episode ist der Dreiecksgeschichte selbst gewidmet. Hier zeigt sich, dass die bisher besprochene Vermischung von Motivationen und Handlungsgründen auch als Einmischung realisierbar ist. Während die Sommerfrischler ohne Frau von Trübezal in der Schwemm'zurück- und aus der Handlung herausbleiben, wird der Ich-Erzähler als Figur aktiv und übernimmt die Rolle des Detektivs im italienischen Fall. Er recherchiert, hält jeweils Unterredungen mit den in Konflikt geratenen Protagonisten und trägt selbst mit dazu bei, dass die Situation eskaliert. Nach Begegnungen und Absprachen mit Franz, Luis und Marcello beteiligt er sich am großen Bekenntnisgespräch Tonios und an der Lüftung der Rachegeschichte. Nachdem nun Marcello dieses Gespräch ausgehorcht und selbst erfahren hatte, dass Tonios Vater an Ridolfos Tod Schuld trägt, beginnt eine verzweifelte Hetzjagd im Wald: man sucht Marcello, damit er zur Vendetta nicht wieder nach Italien abreist, man sucht Tonio, damit er diesen nicht gewaltsam daran verhindert, und letztendlich muss auch Luis, auf der Suche nach den beiden, in den nächtlichen Wald. Hierbei ist der Erzähler gleichzeitig narratives Medium und figuraler Mittelsmann: Er wird gebeten, mehrfach nach Carrara zu telegrafieren und Texte - und seien es recht kurze Texte - über die Sachlage auf diegetischer Ebene zu verfassen; er ist es auch, der sich mit Marcellos Hund versteht - „Es war klar, er lockte mich“ (325) -, der ihn zum verunglückten Besitzer führt. In seiner besonderen Eigenschaft vermag er sogar Tonio zu überreden, den schwer verletzten Todfeind zu bergen, und er nimmt auch Marcello gleichsam eine Beichte ab, womit das letzte Puzzlestück in der italienischen Geschichte gefunden wird. Mit allen diesen Eingriffen kommt es schließlich zur Schlichtung des Konflikts. In seiner narrativ-diegetischen Doppelrolle führt ,Dr. Franz Schneller, Sekretär aus Wien"die Handlung auf ihren Höhepunkt: zur höchsten Konfliktentladung und zum stärksten Verwachsen der drei Gruppen miteinander. Durch figurellen Einsatz zum erzählerischen Zweck prägt sich die Sommerfrischengesellschaft auch sozial endgültig der dörfischen (bzw. der fremdländischen) ein.

Mit den letzten beiden Konfliktepisoden ist freilich viel zu viel offen gelassen, um mit der Abreise des Erzählers ein beruhigendes Ende zu bieten. Dessen bereits 
zitierte Reflexion über ,die Handlung voll mit allen diesen Fäden, ... roten, weißen, grünen“ (351) macht zu Recht klar, dass die kollektiven Angelegenheiten weder mit dem Abgang der Frau von Trübezal noch mit den Bekenntnissen der Figuren bzw. Ereignissen der Dreiecksgeschichte abgeschlossen sind. Insofern haben die beiden als eigenständige Kapitel durchnummerierten und auch umfangreichen - Epiloge „Ein Jahr später“ (352ff) und „Noch ein Jahr später“ (394ff) mehr zu leisten als ein kurzes Fazit und dürfen in dieser Hinsicht als die vierte Konfliktepisode behandelt werden. Die Frage, weshalb es zwei Epiloge gibt, erklärt sich aus genau dem Sachverhalt, dass zwei Konfliktbereiche offengelassen wurden. Entsprechend rundet sich im ersten Epilog nur die Sommerfrischengeschichte ab, während die Dreiecksgeschichte eines weiteren Besuchs des Erzählers im Althof und damit eines weiteren Epilogs bedarf.

Der erste Epilog beginnt mit wiederholter Reflexion darauf, dass im Althof „verwickelte[...] Schicksale“ (352) gewärtigt worden sind. Denn ,[w]o ein Mensch lebt, dort lebt gleich jeder Keim mit, aus dem nur irgend etwas Menschliches sich entwickeln kann“ (353). Bezüglich der Entwicklung der Sommerfrische findet der Erzähler jedenfalls ermutigende Zustände vor: Seit Franz und Luis den Althof übernommen haben, blüht das Geschäft. Was die Gäste angeht, ist nicht nur Frau von Trübezal feierlich versöhnt und 'zurückgewonnen 'worden: „[F]ast alle waren wiedergekommen, die guten Vorjährigen. Dem Rundschreiben des Holzer-Franz, der sie in der Schwemm 'so trefflich unterhalten, war schwer zu widerstehen. “ (375) Im Anklang an den fröhlichen Beginn des Romans kann der Erzähler auch neuere Gäste vorstellen - „Sogar ein Israelit war schon da, was den Franz besonders freute, als Beweis, daß das Publikum » anbiß «.“(376) -, die die Gesamtzahl praktisch verdoppeln. Entsprechend lassen sich auch die neuen Investitionen würdigen (vgl. oben). Insofern erfüllt der Epilog die gewünschte Rolle und führt die 'Geschichte einer Sommerfrische'mehr als zufriedenstellend und humoristisch zu Ende.

Anders verhält es sich mit der Dreiecksgeschichte. Ein weiteres tiefgreifendes Gespräch mit Franz und Beobachtungen über Luis legen klar zutage, dass Tonios Verhaftung und die geschäftsstrategische Eheschließung der beiden Einheimischen noch nicht der glückliche Ausgang sind. Es hat sich noch nicht bewahrheitet, was selbst Marcello - im romantisch-italienischen Modus - wie folgt ausgesprochen hat: „Der Franz und die Luis, das ist wie Ridolfo und Estella, aber oben, oben, im Norden, hinter dem Rücken der Sonne. “ (348) Statt einer Erfüllung dieser Art muss Franz dem Erzähler gegenüber sehr nüchtern argumentieren und es mit einem Hinweis aufs zukünftige Familienglück bewenden lassen Bei aller Sommerfrischenfröhlichkeit des ersten Epilogs bedarf das Liebesglück eines neuen Anlaufs und eines zweiten Epilogs - ,voll der besten Hoffnungen auf die Zukunft, die sich dort [im Althof, E.] freundlich angesponnen“ (393).

„Noch ein Jahr später"geschieht tatsächlich mehr, als das erhoffte Kinderglück die Geburt des Franzl - in den Althof bringen mag. Als eine weitere und letzte Verwicklung der Lebensgeschichten wird die Beziehung zwischen Franz und Luis nochmal durch den italienischen Einfluss berührt. Ein Brief von Holzer informiert den Erzähler darüber, dass Luis die Initiative ergriffen und durch ihre 
Zeugenaussage die Freisprechung Tonios bewirkt hat. ${ }^{16}$ Für den nächsten Besuch des Erzählers im Althof bleiben also weiterhin Fragen offen und auch daselbst scheint nicht alles zum endgültigen Schluss gekommen zu sein.

Ich blieb mehrere Tage in dem gemütlichen kleinen Narrenhause, dessen wirklicher Direktor der Franzl war, und bewunderte den Holzer-Franz als Kinderfrau. Auch hatte ich fortwährend eine eigene Empfindung, als sollte ich noch warten, in dieser verwunschenen Gegend, noch einen Tag, und immer noch einen. Und ich hatte recht. (397)

Die anschließende Szenenfolge erzählt tatsächlich die Wiederkehr des Tonio, die mit Familienkatastrophe droht. Die Wiederholung des früher Geschehenen erweist sich doch im Nachhinein als (Alp-)Traum des Erzählers, zu dem Luis vermerkt: "» Und wenn er [Tonio, E. H.] schon käme! « [...] Der Franz ist stärker als das ganze Königreich Italien.“ (406) Damit bekennt sich Luis zu ihrer neuen-alten Lebenssituation. Ob „Franz“hier ihren Ehemann oder den Franzl meint, bleibt dabei allerdings offengelassen. Aber auch Tonio gibt seinen symbolischen Segen auf den restituierten ländlich-nationalen Status quo: Im kurzen Epilog dieses zweiten Epilogs - in einem zusätzlichen Bericht in der Jetztzeit - beschreibt der Erzähler im Althof „ein Relief aus Carrara-Marmor“ (ebd.), das Luis und Franz und einen segnenden Schutzengel zeigt, der von „überirdischer Schönheit“ (ebd.) ist und der Eingravierung zufolge Estella heißt. Man weiß jetzt endgültig, dass Luis ‘befreiende Tat zu keinem weiteren Konflikt geführt hat und der Künstler Tonio mit diesem Geschenk - „Von Paris ist das Werk gekommen“ (407) - auf die nächste tragische Liebesgeschichte italienisch-österreichischer Art verzichtet. Die ehemaligen Geliebten aus dem Norden und dem Süden haben sich ihrem Schicksal ergeben, wie gut oder schlecht man es auch finden mag. Die bunten Fäden sind jedenfalls wieder entwirrt und der Sommergast kann sich von ihnen ab- und anderweitigen nicht-sommerlichen - Beschäftigungen zuwenden.

\section{„der poetischeste Materialist“ (M. W. 1897, 3)}

In einer zeitgenössischen Bücherschau tut man von Hevesis Die Althofleute als von einem „reizenden, lustig-ernsten Sommerroman“ (Anonym 1898, 4) Erwähnung. Die Charakterisierung als „lustig-ernst"signalisiert dabei Vieldeutigkeit in den Intentionen des Romans. Tatsächlich stellt sich die Frage, ob diese Lektüre 'von Urlaubern - für Urlauber'lediglich für die Unterhaltung konzipiert ist. Entlang der vier Konfliktepisoden zeichnet sich eine Struktur ab, die mit (vor-)gefundener Harmonie (erste Episode: Verspannung und Versöhnung mit abschließendem Fest in der Schwemm') beginnt und in Zerfall (zweite Episode: Frau von Trübezals Abgang) bzw. Zerwürfnis (dritte Episode: Bekenntnisse und Auseinandersetzung der Italiener) mündet. Epilogisch werden diese Konflikte zwar geschlichtet (vierte

\footnotetext{
16 Über den Fall Ridolfo erfährt man in den Epilogen übrigens auch aus Zeitungsberichten, die 'wörtlich' zitiert werden und voll falscher Angaben sind. Eine Persiflage - des Feuilletonisten Hevesi! - auf die Nachrichtenerstattung, zugleich eine Karikatur auf das eigene narrative Verfahren, mit halbwegs zutreffenden Informationen - etwa mit einem 'Sankt Ruprecht'- zu operieren.
} 
Episode: Wiederaufleben der Sommerfrische mit Franz und Luis als Paar), aber auch mit keinem Idyll abgeschlossen. Die anfänglich unbeholfen-spontan entstandene Sommerfrische wird professionalisiert - man braucht nicht mehr alle Gäste persönlich zu kennen -, und das Ehepaar schließt eine Vernunftehe ab. Die Unberührtheit des Ortes und die schrankenlose - soziale bzw. nationale Grenzen überschreitende - Liebe werden verabschiedet und die 'Parteien'(auch die liebenden 'Parteien') einem Kompromiss zugeführt. Die Natur lässt sich auch in kleinen, komfortablen Zügen mit Vollpension genießen. Und die Liebe kommt zur Ehe nachträglich dazu. Insofern ist der Roman auch eine Geschichte der verlorenen Unschuld (der Landschaft und der Seele) - eine romantische Geschichte, die in realistische Lebensweisheit eingefasst und rückwirkend relativiert wird. Man beobachtet mit dem Erzähler, wie sich das Ländliche dem Städtischen ergibt, und parallel dazu auch, ,wie diese beiden guten Menschen [Luis und Franz, E. H.] den guten Krieg miteinander führten, in dem jeder Sieger auch Besiegter ist und jeder Besiegte auch Sieger“ (383). Man sieht, dass auch in der Sommerfrische lebensstrategische Entscheidungen getroffen werden und Zusammenleben ausgehandelt wird. Der Roman demonstriert dies, indem er die Geschichte des Ortes mit der Geschichte der Menschen kombiniert. Dadurch wird dem 'sommerlichen'Stoff eine sozialhistorische und -psychologische Tiefendimension verliehen.

Sommerfrischen sind Heterotopien, in dieser Eigenschaft jedoch nicht außerhalb der Gesellschaft zu verorten. ${ }^{17}$ Vielmehr sind sie Laboratorien des Sozialen: Schauplatz von (Selbst-)Begegnungen, die ansonsten nicht stattfinden würden und von Konfliktzonen, in denen die Konsequenzen ausgetragen werden. Hevesis Versuch, dieses historische Feld in Handlungssträngen eines „Sommerromans"umzusetzen, ist etwas Besonderes sowohl in seinem Schaffen als auch in der Literatur der Sommerfrischen. Hier werden die gängigen Urlauber-Feuilletons und -berichte, die Dorfgeschichte und natürlich auch die zeitungsübliche Trivialliteratur genutzt, umgewandelt und auch überboten. Dass sich Hevesi ,,mit seiner prächtigen guten Laune nie von dem Ernst der Situation unterkriegen [läßt] "und statt fragmentarisierender Modernität letztendlich „das beruhigendste Sicherheitsgefühl“ (M. W 1897, 3) vermittelt, gehört zu seinem Profil als Feuilletonist, der seinen Text - bei welchem Thema auch immer - letztlich 'rund', bündig und zeitig abzuschließen hat.

\section{Literaturverzeichnis}

Anonym. (1898). Aus Christkindl's Bücherkasten. Literarische Weihnachtsplauderei II. Innsbrucker Nachrichten, 14. Dezember 1898, S. 4.

Foucault, M. (1992). Andere Räume (Übers., W. Seitter). In K. Barck u.a. (Hg.): Aisthesis. Wahrnehmung heute oder Perspektiven einer anderen Ästhetik (S. 34-46). Leipzig: Reclam.

Haas, H. (1992). Die Sommerfrische - Ort der Bürgerlichkeit. In H. Stekl u. a. (Hg.): „Durch Arbeit, Besitz, Wissen und Gerechtigkeit“. Bürgertum in der Habsburgermonarchie II (S. 364-377). Wien; Köln; Weimar: Böhlau.

17 Vgl. Foucault 1992, 39. 
Hevesi, L. (1897). Die Althofleute. Ein Sommerroman (mit Illustrationen von W. Schulz). Stuttgart: Adolf Bonz \& Comp.

Illustrierter Wegweiser der österreichischen Kurorte, Sommerfrischen und Winterstationen. (1909). Heft: Steiermark. Wien/Leipzig: Elbemühl/K. k. Hoftheater-Druckerei.

Kos, W. (1995). Riten der Geborgenheit. Wenn Landschaft zum schönen Zimmer wird. In E. Pusch, M. Schwarz (Hg.): Architektur der Sommerfrische (S. 7-20). St. Pölten, Wien: Niederösterreichisches Pressehaus.

Lippmann, H.-Chr. (2016). Sommerfrische als Symbol- und Erlebnisraum bürgerlichen Lebensstils. Zur gesellschaftlichen Konstruktion touristischer ländlicher Räume. Dissertation zur Erlangung des akademischen Grades Doktor der Philosophie. Fakultät VI - Planen Bauen Umwelt der Technischen Universität Berlin.

M. W. (1897). Literatur (Ludwig Hevesi - Ludwig Ganghofer - Johannes Ziegler.). Wiener Zeitung, 15. Jänner 1897, S. 3-4.

Mai, A. (2004). Touristische Räume im 19. Jahrhundert. Zur Entstehung und Ausbreitung von Sommerfrischen. WerkstattGeschichte, 36, 7-23.

Michelis, A. (1869). Reiseschule für Touristen und Curgäste. Leipzig: Adolf Gumprecht. http://www. deutschestextarchiv.de/book/show/michelis_reiseschule_1869 [24.05.2017].

Plath, J. (Ed.). (1999a). Sommerfrische. Ein literarisches Lesebuch. München: Deutscher Taschenbuch Verlag.

Plath, J. (1999b). Nachwort. In Plath (Hg.) 1999a, 263-272.

Teuber, O. (1886). Auf der Sonnenseite. Fremden-Blatt, 19. Oktober 1886, 11.

Traeger, A. (1897). Ein humoristischer Roman. Neue Freie Presse, 15. April 1897, S. 1-2.

Tworek, E. (Ed.). (2011). Literarische Sommerfrische. Künstler und Schriftsteller im Alpenvorland. Ein Lesebuch. München: Allitera. 